\title{
Oxygen Uptake at Critical Speed and Power in Running: Perspectives and Practical Applications
}

\author{
Aurélien Patoz, Thomas Blokker, Nicola Pedrani, Romain Spicher, Fabio Borrani, \\ and Davide Malatesta
}

\begin{abstract}
Purpose: Intensity domains are recommended when prescribing exercise, and critical power/speed (CP/CS) was designated the "gold standard" when determining maximal metabolic steady state. CS is the running analog of CP for cycle ergometry. However, a CP for running could be useful for controlling intensity when training in any type of condition. Therefore, this study aimed to estimate external, internal, and total $\mathrm{CP}\left(\mathrm{CP}_{\text {ext }}, \mathrm{CP}_{\text {int }}\right.$, and $\left.\mathrm{CP}_{\text {tot }}\right)$, obtained based on running power calculations, and verified whether they occurred at the same percentage of peak oxygen uptake as the usual CS. Furthermore, this study examined whether selecting strides at the start, half, or end of the exhaustive runs to calculate running power influenced the estimation of the 3 CPs. Methods: Thirteen male runners performed a maximal incremental aerobic test and 4 exhaustive runs $(90 \%, 100 \%, 110 \%$, $120 \%$ peak speed) on a treadmill. The estimations of CS and CPs were obtained using a 3-parameter mathematical model fitted using weighted least square. Results: $\mathrm{CS}$ was estimated at $4.3 \mathrm{~m} / \mathrm{s}$ while the estimates of $\mathrm{CP}_{\mathrm{ext}}, \mathrm{CP}_{\text {int }}$, and $\mathrm{CP}_{\text {tot }}$ were 5.2, 2.6, and $7.8 \mathrm{~W} / \mathrm{kg}$, respectively. The corresponding $\mathrm{VO}_{2}$ for CS was 82.5 percentage of peak oxygen uptake and 81.3, 79.7, and 80.6 percentage of peak oxygen uptake for $\mathrm{CP}_{\mathrm{ext}}, \mathrm{CP}_{\mathrm{int}}$, and $\mathrm{CP}_{\text {tot }}$, respectively. No systematic bias was reported when comparing CS and $\mathrm{CP}_{\mathrm{ext}}$, as well as the 3 different $\mathrm{CPs}$, whereas systematic biases of $2.8 \%$ and $1.8 \%$ were obtained for the comparison among $\mathrm{CS}$ and $\mathrm{CP}_{\text {int }}$ and $\mathrm{CP}_{\text {tot }}$, respectively. Nonetheless, the $\mathrm{VO}_{2}$ for $\mathrm{CS}$ and $\mathrm{CPs}$ were not statistically different $(P=.09)$. Besides, no effect of the time stride selection for CPs as well as their resulting $\mathrm{V}_{2}$ was obtained $(P \geq .44)$. Conclusions: The systematic biases among $\mathrm{VO}_{2}$ at $\mathrm{CS}$ and $\mathrm{CP}_{\text {int }}$ and $\mathrm{CP}_{\text {tot }}$ were not clinically relevant. Therefore, $\mathrm{CS}$ and $\mathrm{CPs}$ closely represent the same fatigue threshold in running. The knowledge of $\mathrm{CP}$ in running might prove to be useful for both athletes and coaches, especially when combined with instantaneous running power. Indeed, this combination might help athletes controlling their targeted training intensity and coaches prescribing a training session in any type of condition.
\end{abstract}

Keywords: hyperbolic model, mechanical work, intensity domains, fatigue, critical intensity

The importance of exercise intensity in training adaptations is well established. ${ }^{1}$ The intensity is often prescribed based on the percentage of maximal oxygen uptake $\left(\mathrm{V}{ }_{2}\right.$ max $)$ or maximal heart rate. ${ }^{1-3}$ However, due to a large intersubject variability, the recommendation of exercise intensity based on these parameters has been criticized by several authors. ${ }^{4-6}$ Indeed, inequivalent metabolic responses were obtained for the same given percentage. Instead, exercise intensity domains have been recommended ${ }^{7}$ and shown to trigger targeted adaptations. ${ }^{8}$ Among several possibilities, ${ }^{9-12}$ critical power/speed (CP/CS) ${ }^{11-14}$ can be used, which is described as the highest power/speed output at which metabolic homeostasis is achieved, ${ }^{15}$ and may be considered the most important fatigue threshold in exercise physiology. ${ }^{16}$

The CP/CS is considered a better individualization method for training, provides a useful insight in the best possible performance for a given work/distance and power/speed for athletes, ${ }^{17}$ and permits the separation of heavy from severe intensity domains. ${ }^{12,15}$ For this reason, the calculation of CP/CS was designated the "gold standard" when determining maximal metabolic steady state. ${ }^{12} \mathrm{CP} / \mathrm{CS}$ is usually obtained by the typical CP/CS test, that is, several exhaustive cycling/ running tests between $90 \%$ and $130 \% \dot{\mathrm{VO}}_{2}$ max. $^{18}$

The authors are with the Inst of Sport Sciences, University of Lausanne, Lausanne, Switzerland. Patoz is also with the Research and Development Dept, Volodalen Swiss Sport Lab, Aigle, Switzerland. Patoz (aurelien.patoz@unil.ch) is corresponding author.
The CS is considered as the running analog of $\mathrm{CP}$ for cycle ergometry. ${ }^{18}$ However, speed could become a nonrelevant metric for separating between intensity domains, for example, when training on a variable terrain with uphill, flat, and downhill parts, or in a very windy condition, where air resistance starts playing an important role. In these cases, controlling running power sounds more suitable. Such metrics are available from several commercial power meters (eg, Runscribe [Scribe Lab Inc, Half Moon Bay, CA], Stryd [Stryd Inc Boulder, CO], or Myotest [Myotest SA, Sion, Switzerland]), which are based on inertial measurement units. ${ }^{19}$ Nonetheless, training that targets a specific power-based intensity domain requires an estimation of a running CP. Fortunately, performing the $\mathrm{CP} / \mathrm{CS}$ test on an instrumented treadmill provides the measure of ground reaction forces, which allow calculating positive external work $\left(W_{\text {ext }}\right)$ and thus external power $\left(P_{\text {ext }}\right) \cdot{ }^{20}$ Besides, internal power $\left(P_{\text {int }}\right)$ can be derived from the equation proposed by Nardello et $\mathrm{al}^{21}$ and summing these 2 leads to the total power $\left(P_{\text {tot }}\right)$. Each of these 3 different powers could be used to estimate a corresponding running $\mathrm{CP}: \mathrm{CP}_{\text {ext }}, \mathrm{CP}_{\text {int }}$, and $\mathrm{CP}_{\text {tot }}$. These calculations had, to the best of our knowledge, never been investigated so far, therefore constituting the first aim of this study. Moreover, this study verified whether CPs and CS were at the same physiological state, for example, the same percentage of peak $\mathrm{VO}_{2}\left(\% \dot{V}_{2}\right.$ peak). As it sounds mechanically logical, we hypothesized that (1) each CP and CS would occur at the same $\% \mathrm{VO}_{2}$ peak, that is, no systematic bias would be reported between $\dot{\mathrm{V}} \mathrm{O}_{2}\left(\% \dot{\mathrm{VO}}_{2}\right.$ peak $)$ at $\mathrm{CS}$ and at each of the 3 different CPs. Moreover, this would also be true for the different pairs of CPs 
because $\dot{\mathrm{VO}}_{2}$ at $\mathrm{CP}$ represents a specific metabolic rate (ie, specific parameter of aerobic function) achieved at different combinations of $P_{\text {ext }}$ and $P_{\text {int }}$ outputs as previously reported in cycling at different pedaling frequency. ${ }^{22}$ Therefore, we hypothesized that (2) no systematic bias would be reported among $\dot{\mathrm{VO}}_{2}$ at the 3 different CPs. 23

Although CP/CS technically ought to be a defined precise value, research has established that determining that value may not be as accurate as theoretical beliefs. ${ }^{23-27}$ More specifically, a day-to-day intrasubject variability of up to $5 \%$ to $6 \%$ when defining $\mathrm{CP} / \mathrm{CS}$ was highlighted. ${ }^{23}$ In addition, a decrease of $\mathrm{CP}$ was obtained when it was assessed using a 3-minute all-out test (up to 10\%) on a cycle ergometer following a prolonged (120 min) submaximal exercise. ${ }^{25}$ Besides, a decrease in the internal work $\left(W_{\text {int }}\right)$, due to a decrease of both stride frequency and contact time, was observed close to the end of an exhaustive run at a speed corresponding to $95 \% \dot{\mathrm{VO}}_{2} \mathrm{max}^{24}$ Similarly, Candau et $\mathrm{al}^{26}$ observed a slight decrease of the step frequency at the end of an exhaustive treadmill run performed at a speed corresponding to the participants' personal record over $3000 \mathrm{~m}$, thus supporting the idea of a decrease in $W_{\text {int }}$. On the contrary, Avogadro et $\mathrm{al}^{27}$ obtained no difference in $W_{\text {int }}$ at the third and last minute of an exhaustive run at a speed corresponding to $90 \%$ $\dot{\mathrm{VO}}_{2} \max$. As for $W_{\text {ext }}$, no change during the exhaustive run was reported by Borrani et $\mathrm{al}^{24}$ and by Avogadro et al, ${ }^{27}$ while Candau et $\mathrm{al}^{26}$ observed its increase at the end of the run. Therefore, these results depict conflicting evidence in the scientific literature about the changes in $W_{\text {ext }}$ and $W_{\text {int }}$, and thus in total work $\left(W_{\text {tot }}\right)$, through the time course of an exhaustive run. Moreover, it might be that a different exercise intensity, that is, a supramaximal intensity, is also affecting the changes in $W_{\text {ext }}, W_{\text {int }}$, and $W_{\text {tot }}$ through the exhaustive run. Altogether, this could ultimately lead to different estimations of $\mathrm{CP}_{\text {int }}, \mathrm{CP}_{\text {ext }}$, and $\mathrm{CP}_{\text {tot }}$ when calculated using strides selected at different time points of the exhaustive run. Hence, the second aim of this study was to examine whether selecting strides at the start, half, and end of the exhaustive runs influenced the estimation of the 3 different CPs. We hypothesized that (3) time stride selection would influence the estimation of the 3 different CPs.

\section{Methods}

\section{Subjects}

Thirteen male runners gave written informed consent to participate in the present experiment (age 25.7 [4.4] y; height 179 [5] cm; body mass 68 [5] kg). For study inclusion, participants were required to be in good self-reported general health with no symptoms of cardiovascular disease or major coronary risk factors, no current or recent lower-extremity injury that could prevent them from giving $100 \%$ of their capacity during the test, and to meet a certain level of running performance. More specifically, runners were required to have a speed associated with $\dot{\mathrm{VO}}_{2}$ max greater or equal to $4.44 \mathrm{~m} / \mathrm{s}(16 \mathrm{~km} / \mathrm{h})$. The study protocol was approved by the Commission cantonale d'éthique de la recherche sur l'être humain (CER-VD 2018-01814) and adhered to the latest Declaration of Helsinki of the World Medical Association.

\section{Design}

Each participant completed 5 experimental sessions interspersed by at least 2 days in the laboratory. All participants were advised to avoid strenuous exercise the day before a test but to maintain their usual training program otherwise. During the first session, participants completed a maximal incremental aerobic test on an instrumented treadmill (Arsalis T150-FMT-MED; Arsalis, Louvain-la-Neuve, Belgium). This test consisted of a 10-minute warm-up at $2.78 \mathrm{~m} / \mathrm{s}$ followed by an incremental increase in the running speed of $0.28 \mathrm{~m} / \mathrm{s}$ every 2 minutes until exhaustion. Throughout the test, participants breathed into a mask connected to a gas analyzer (Quark; COSMED, Rome, Italy). Pulmonary gas exchange variables (expired minute ventilation, $\dot{\mathrm{VO}}_{2}$, and carbon dioxide output) were measured breath-by-breath and subsequently averaged over 10 -second intervals throughout the test. Before each test, the $\mathrm{O}_{2}$ and $\mathrm{CO}_{2}$ analyzers were calibrated using room air and known concentrations of calibration gas $\left(16.00 \% \mathrm{O}_{2}, 5.02 \% \mathrm{CO}_{2}\right.$, and balanced $\mathrm{N}_{2}$ ), and the turbine was calibrated using a $3-\mathrm{L}$ syringe (Hans Rudolph, Shawnee, KS).

This test was used, first, to determine the peak speed (PS) of the maximal incremental aerobic test of each participant. PS is defined as the running speed of the last fully completed increment $\left(s_{\text {last-inc }}\right)$ plus the fraction of time spent in the following uncompleted increment $(\alpha)$ multiplied by the running speed increment $(\Delta s=0.28 \mathrm{~m} / \mathrm{s})^{28}:$ PS $=s_{\text {last-inc }}+\alpha \Delta s$. Second, the $\dot{\mathrm{VO}}_{2}$ peak was defined as the highest measured $\mathrm{VO}_{2}$.

The other 4 tests were performed in a randomized order and consisted of exhaustive runs at a given percentage of the participant's PS $(90 \%, 100 \%, 110 \%$, and 120\%). These tests were as follows: after a 10-minute warm-up at $2.78 \mathrm{~m} / \mathrm{s}$ and a 5 -minute rest period, the running speed was increased to a given percentage of PS, and the participant had to maintain the pace until exhaustion. The time to exhaustion was collected for each of the 4 sessions. No information about the timings or running speed was given to any of the participants, who were strongly encouraged, during any of the 5 experimental sessions. All participants were familiar with running on a treadmill.

Ground reaction forces $(1000 \mathrm{~Hz})$ were collected using the force plate embedded into the treadmill during the last 30 seconds of each minute passed in each of the 4 exhaustive runs, as well as in the maximal incremental aerobic test. Forces were subsequently lowpass filtered at $20 \mathrm{~Hz}$ using a fifth-order Butterworth filter. From these data, 10 successive strides were selected and chosen to be at the first (start), middle (half), and last (end) minute of each exhaustive run and at every 2 minutes (corresponding to the timing of the speed increment) of the maximal incremental aerobic test. This allowed assessing running biomechanics. More specifically, $W_{\text {ext }}$, that is, the sum of positive potential and kinetic works, ${ }^{20} W_{\text {int }},{ }^{21}$ and $W_{\text {tot }}$ were computed (in joule per kilogram per meter) using the 3-D force plate software (Arsalis), which further facilitated obtaining $P_{\text {ext }}, P_{\text {int }}$, and $P_{\text {tot }}$ (ie, by multiplying work with running speed).

\section{Methodology}

The estimations of CPs and CS were obtained from the 3-parameter model formulation proposed by Morton, ${ }^{29}$ that is, by expressing power $\left(P_{\text {ext }}, P_{\text {int }}\right.$, and $\left.P_{\text {tot }}\right) /$ speed as function of time:

$$
\begin{gathered}
P(t)=\mathrm{CP}+\frac{\mathrm{W}^{\prime}}{t+\frac{\mathrm{W}^{\prime}}{P_{\max }-C S}}, \\
s(t)=\mathrm{CS}+\frac{d^{\prime}}{t+\frac{d^{\prime}}{s_{\max }-\mathrm{CS}}},
\end{gathered}
$$

where $P_{\max } / s_{\max }$ are the maximal instantaneous power/running speed. However, time being the dependent variable, error minimization was performed on this variable, that is, on the $x$-axis, ${ }^{30}$ using 
weighted least square (iteratively using Levenberg-Marquardt algorithm) $)^{31,32}$ with weights proportional to the inverse of the time to exhaustion, as suggested by Morton and Hodgson. ${ }^{18}$

To obtain the $\mathrm{VO}_{2}$ at the CS estimates for each participant, first the $\dot{\mathrm{V}}_{2}$ recorded during the maximal incremental aerobic test was averaged during the last 30 seconds of each 2-minute increment. Then, a linear relation between these $\dot{\mathrm{VO}}_{2}$ 's and corresponding running speeds was constructed. Finally, the $\mathrm{VO}_{2}$ at the CS estimates was simply given by placing these CSs on the previously computed linear regressions. Similarly, to obtain the $\dot{\mathrm{VO}}_{2}$ at the $\mathrm{CP}$ estimates for each participant, a linear regression of $\dot{\mathrm{VO}}_{2}$ as function of power (both obtained during the maximal incremental aerobic test) was computed. Then, the $\dot{\mathrm{VO}}_{2}$ at the $\mathrm{CP}$ estimates were given by inserting these CPs into the previously mentioned linear regressions. Data analysis was performed using Python (version 3.7.4; Python Software Foundation. available at http:// www.python.org).

\section{Statistical Analysis}

All data are presented as mean (SD). Comparison among the $\mathrm{VO}_{2}$ at CS and CPs calculated using strides selected at the start of the exhaustive runs and between pairs of CPs were performed using a Bland-Altman analysis. Corresponding 95\% confidence intervals were calculated. Besides, after having inspected residual plots and having observed no obvious deviations from homoscedasticity and normality, these $\mathrm{VO}_{2}$ 's were compared using 1-way repeatedmeasures analysis of variance (RM-ANOVA) with Mauchly correction for sphericity and employing Holm corrections for pairwise post hoc comparisons. Then, 1-way RM-ANOVA were used to investigate the effect of the time stride selection (start, half, and end) on the estimation of $\mathrm{CP}_{\text {ext }}, \mathrm{CP}_{\text {int }}$, and $\mathrm{CP}_{\text {tot }}$, and their corresponding $\mathrm{VO}_{2}$. Finally, the effect of the time stride selection and percentage of PS (\%PS) on the calculation of $P_{\text {ext }}, P_{\text {int }}$, and $P_{\text {tot }}$ were investigated using 2-way ([start, half, end time stride selection] $\times$ [90\%PS, 100\%PS, 110\%PS, 120\%PS]) RM-ANOVA. Statistical analysis was performed using Jamovi (version 1.6.18; [computer software], retrieved from https://www.jamovi.org) with a level of significance set at $P \leq .05$.

\section{Results}

The variables determined by the maximal incremental aerobic test were $\dot{\mathrm{VO}}{ }_{2}$ peak: $64.2(4.2) \mathrm{mL} / \mathrm{min} / \mathrm{kg}, \mathrm{S}_{\text {last-inc }}: 5.2(0.3) \mathrm{m} / \mathrm{s}$, and PS: $5.3(0.3) \mathrm{m} / \mathrm{s}$. The average $R^{2}$ obtained for the linear regression of the $\dot{\mathrm{VO}}_{2}$ as a function of time relationship recorded during the maximal incremental aerobic test was 0.98 (0.03), while those for the linear regressions of $P_{\text {ext }}, P_{\text {int }}$, and $P_{\text {tot }}$ as function of $\dot{\mathrm{VO}}_{2}$ were 0.97 (0.04), 0.95 (0.05), and 0.97 (0.04), respectively.

The regression analyses for one representative participant and for the 3 powers as function of time, speed as function of time, $\dot{\mathrm{VO}}_{2}$ as function of time of the maximal incremental aerobic test, and powers as function $\dot{\mathrm{VO}}_{2}$ recorded during the maximal incremental aerobic test are presented in Figure 1, together with the $\mathrm{VO}_{2}$ corresponding to the $3 \mathrm{CPs}$ as well as CS.

Bland-Altman plots comparing the $\dot{\mathrm{VO}}_{2}\left(\% \dot{\mathrm{VO}}{ }_{2}\right.$ peak $)$ at $\mathrm{CS}$ with the one at the 3 CPs calculated using strides selected at the start of the exhaustive runs and between pairs of CPs are depicted in Figures 2 and 3, respectively.

No systematic bias (the zero line lies between the $95 \%$ confidence interval) was reported when comparing $\mathrm{CS}$ and $\mathrm{CP}_{\text {ext }}$, that is, $1.1 \%(-1.0 \%$ to $3.3 \%)$, whereas small systematic biases of
$2.8 \%(95 \% \mathrm{CI}, 1.2 \%$ to $4.3 \%)$ and $1.8 \%(0.6 \%$ to $3.0 \%)$ were obtained for the comparison among $\mathrm{CS}$ and $\mathrm{CP}_{\text {int }}$ and $\mathrm{CP}_{\text {tot }}$, respectively. No significant systematic biases were reported when comparing the different $\mathrm{CPs}$, that is, $\mathrm{CP}_{\text {ext }}$ versus $\mathrm{CP}_{\text {int }}$ : $1.6 \%(-1.1 \%$ to $4.3 \%) ; \mathrm{CP}_{\text {tot }}$ versus $\mathrm{CP}_{\text {ext }}:-0.7 \%$ (-2.4\% to $\left.0.9 \%\right)$; and $\mathrm{CP}_{\text {tot }}$ versus $\mathrm{CP}_{\text {int }}: 0.9 \%(-0.2 \%$ to $2.0 \%)$. The 1 -way $\mathrm{RM}-$ ANOVA reported no significant difference among the $\mathrm{VO}_{2}$ for CS and CPs at the start $(P=.09$; Table 1$)$.

The CS and CPs estimated using strides recorded at the start, half, and end of the exhaustive runs as well as their corresponding $\dot{\mathrm{VO}}_{2}\left(\% \dot{\mathrm{VO}}{ }_{2}\right.$ peak $)$ are given in Table 1 . There was no main effect of the time stride selection when estimating the $3 \mathrm{CPs}$ as well as for their resulting $\dot{\mathrm{VO}}_{2}(P \geq .44)$.

Table 2 depicts the time to exhaustion corresponding to the 4 exhaustive runs performed at $90 \%, 100 \%, 110 \%$, and $120 \%$ of the participant's PS as well as $P_{\text {ext }}, P_{\text {int }}$, and $P_{\text {tot }}$ computed at the start, half, and end of the exhaustive runs. There was no significant main effect of the time stride selection on the calculation of the 3 powers $(P \geq .11)$, while there was a significant main effect of $\%$ PS $(P<.001)$, with each of the 3 powers being statistically higher at a higher \% PS than at previous one, as reported by post hoc tests $(P<.001)$. A significant interaction effect was obtained only for $P_{\text {tot }}(P=.03)$, leading to a statistically higher $P_{\text {tot }}$ at the end than at the start $(P=.03)$ and half $(P=.05)$ for $110 \% \mathrm{PS}$ as well as a statistically higher $P_{\text {tot }}$ for all comparisons between a higher $\%$ PS and a lower one $(P<.001 ; 54$ comparisons $)$.

\section{Discussion}

Conventional statistical approaches demonstrated no systematic bias between the $\mathrm{VO}_{2}$ at $\mathrm{CS}$ and $\mathrm{CP}_{\text {ext }}$, whereas systematic biases were obtained among the $\dot{\mathrm{VO}}_{2}$ at $\mathrm{CS}$ and at $\mathrm{CP}_{\text {int }}$ and $\mathrm{CP}_{\text {tot }}$, which partly refuted the first hypothesis. In accordance with the second hypothesis, no systematic biases were reported among the $\mathrm{VO}_{2}$ at the 3 different CPs. Besides, the $4 \mathrm{VO}_{2}$ 's (at $\mathrm{CS}, \mathrm{CP}_{\text {ext }}, \mathrm{CP}_{\text {int }}$, and $\mathrm{CP}_{\text {tot }}$ ) were not statistically different. In addition, this study observed no effect of the time stride selection when estimating $\mathrm{CPs}$, their resulting $\mathrm{VO}_{2}$, as well as the underlying powers used for the CPs calculations, which refuted the third hypothesis.

On the one hand, the $\mathrm{VO}_{2}$ at CS and CPs were not statistically different. Moreover, no systematic bias was reported between the $\dot{\mathrm{VO}}_{2}$ at $\mathrm{CS}$ and $\mathrm{CP}_{\text {ext }}$. However, systematic biases were obtained among the $\dot{\mathrm{VO}}_{2}$ at $\mathrm{CS}$ and $\mathrm{CP}_{\text {int }}$ and $\mathrm{CP}_{\text {tot }}$ (Table 1 and Figure 2). Nonetheless, these differences were reasonably small, that is, smaller than 3\%, and thus not clinically relevant. Furthermore, these differences might be explained by the accuracy of the linear regressions used to estimate the relation between $\dot{\mathrm{VO}}_{2}$ and running speed as well as between $\dot{\mathrm{VO}}_{2}$ and each of the 3 different powers. In fact, even though the $R^{2}$ were quite high ( $\geq .95$ [.05]), they were not perfect and could have led to these small discrepancies. These differences might also be explained by the mathematical model employed to estimate CS and CPs (Equation 1). Indeed, these estimations were based on a model simplifying a more complex system, hence necessarily introducing some errors. In addition, the highest difference was obtained between the $\dot{\mathrm{VO}} 2$ at $\mathrm{CS}$ and $\mathrm{CP}_{\text {int }}$, which might be explained by the fact that $P_{\text {int }}$ was calculated using a model equation ${ }^{21}$ and not using the motion of the body segments relative to the center of mass, further introducing some errors. Therefore, these results suggest that CS and CPs closely represent the same fatigue threshold in running. Nonetheless, the message of Jones et al, ${ }^{33}$ which states that CS and CP should not be grouped together under a nebulous "critical intensity" term but that the 

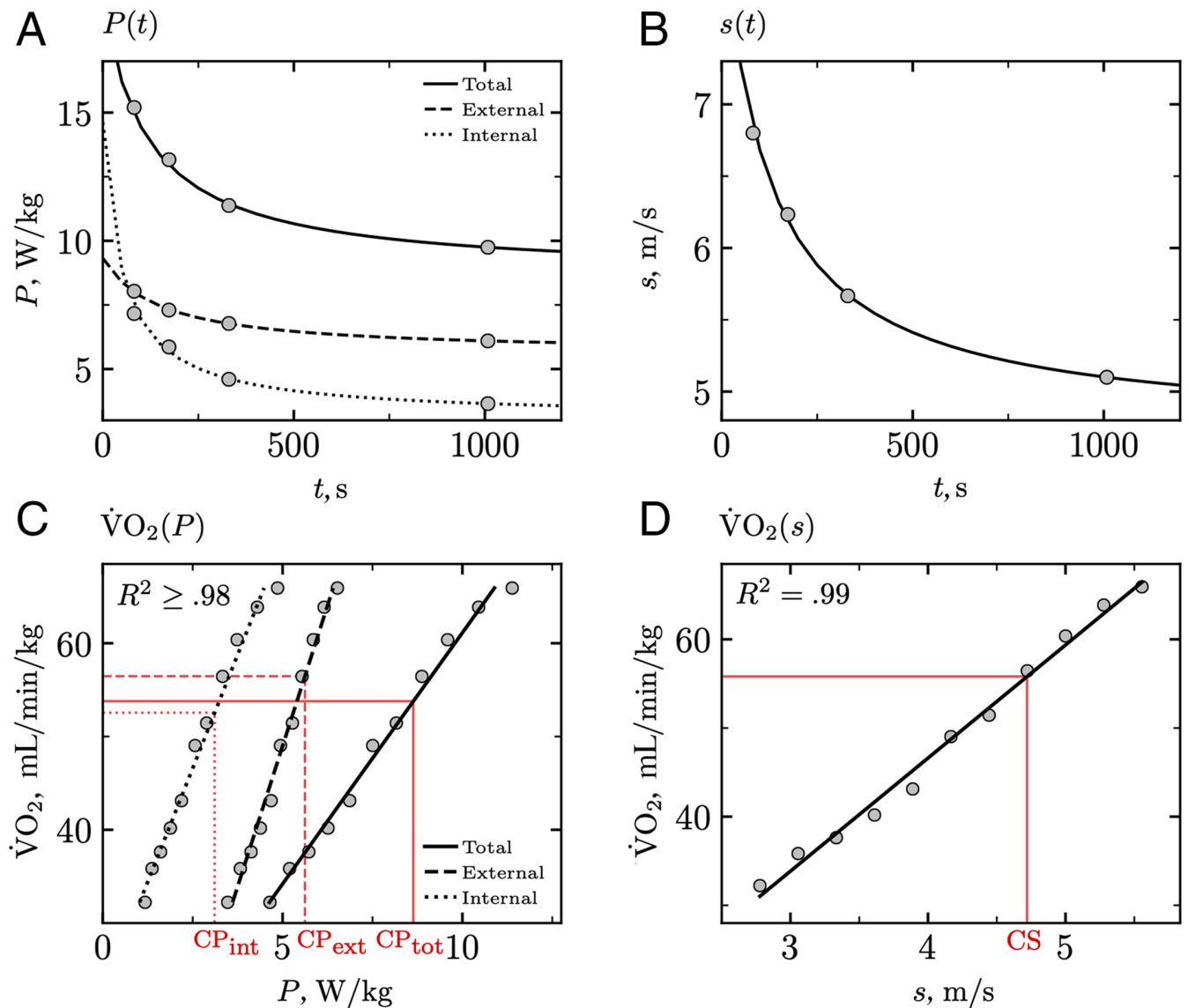

Figure 1 - Regression analyses of 1 representative participant for (A) the 3 different $P_{\text {ext }}, P_{\text {int }}$, and $P_{\text {tot }}$ as function of $t$ obtained using powers calculated using strides selected at the start of the time trials and corresponding times to exhaustion (3-parameter model); (B) $s$ as function of time obtained using fixed speeds (90\%PS, 100\%PS, $110 \% \mathrm{PS}$, and $120 \% \mathrm{PS}$ ) and corresponding times to exhaustion (3-parameter model); (C) $\dot{\mathrm{VO}}_{2}$ averaged during the last 30 seconds of each 2-minute increment of the maximal incremental aerobic test as function of corresponding running speed (linear regression); and (D) $\mathrm{VO}_{2}$ averaged during the last 30 seconds of each 2-minute increment of the maximal incremental aerobic test as function of corresponding powers calculated using strides selected at every 2-minutes of the maximal incremental aerobic test (linear regression). In addition, $\dot{\mathrm{VO}}_{2}$ corresponding to internal, external, and total critical powers $\left(\mathrm{CP}_{\text {int }}, \mathrm{CP}_{\text {ext }}\right.$, and $\left.\mathrm{CP}_{\text {tot }}\right)$ as well as $\mathrm{CS}$ are depicted in $(\mathrm{C})$ and (D), respectively. $\mathrm{CP}$ indicates critical power; $\mathrm{CP}$ ext, external CP; $\mathrm{CP}_{\text {int }}$, internal CP; $\mathrm{CP}_{\text {tot }}$, total CP; CS, critical speed; $P$, power, $P_{\text {ext }}$, external $P ; P_{\text {int }}$, internal $P ; \mathrm{PS}$, peak speed; $P_{\text {tot }}$, total $P ; s$, speed; $t$, time; $\dot{\mathrm{V}} \mathrm{O}_{2}$, oxygen uptake.
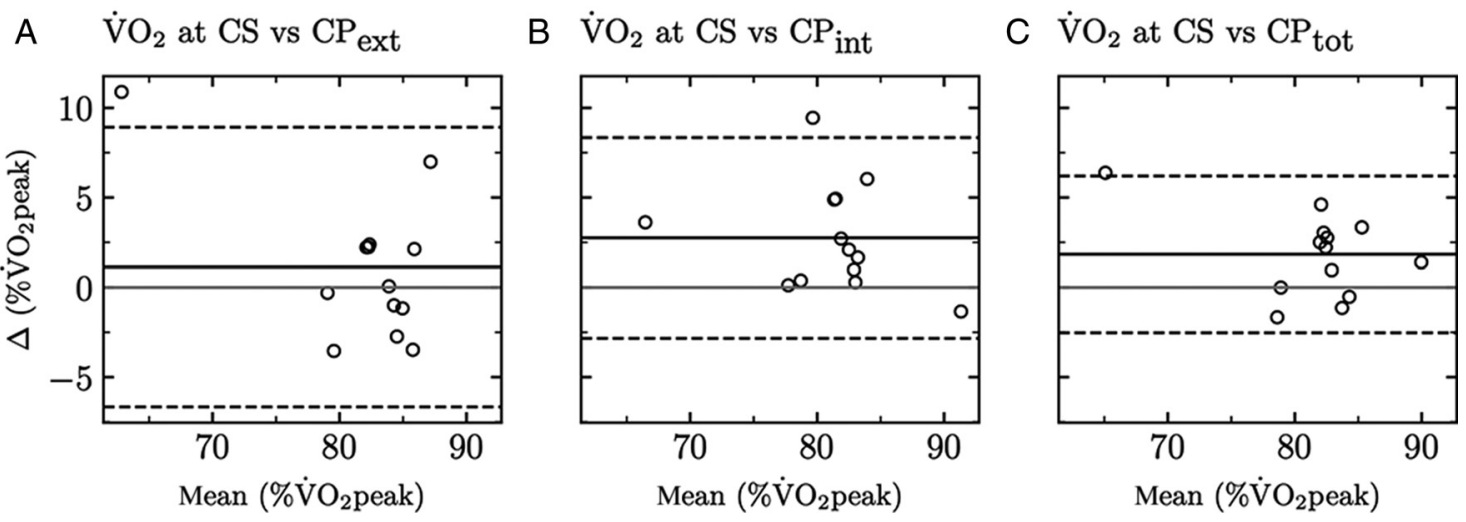

Figure 2 - Bland-Altman plots comparing $\dot{\mathrm{VO}}_{2}$ (expressed as a $\% \dot{\mathrm{VO}}_{2}$ peak) at $\mathrm{CS}$ with (A) $\mathrm{CP}_{\text {ext }}$, (B) $\mathrm{CP}_{\text {int }}$, and (C) $\mathrm{CP}_{\text {tot }}$. $\mathrm{CP}_{\text {indicates critical power; }}$ $\mathrm{CP}_{\text {ext }}$, external CP; $\mathrm{CP}_{\text {int }}$, internal $\mathrm{CP} ; \mathrm{CP}_{\text {tot }}$, total $\mathrm{CP} ; \mathrm{CS}$, critical speed; $\dot{\mathrm{VO}}_{2}$, oxygen uptake; $\% \dot{\mathrm{VO}}_{2}$ peak, percentage of peak rate of oxygen uptake.

proper term (CS, CP, critical force, critical tension, or critical torque) should be used depending on the corresponding measured quantity, must continue to spread. Indeed, the similarity between CS and CPs reported in the present study may only be true because
CS and CPs were both determined during running and thus represent a specific intensity threshold for the same exercise mode. On the contrary, CS and CP assessed during 2 different exercise modes (ie, running and cycling, respectively) do not 

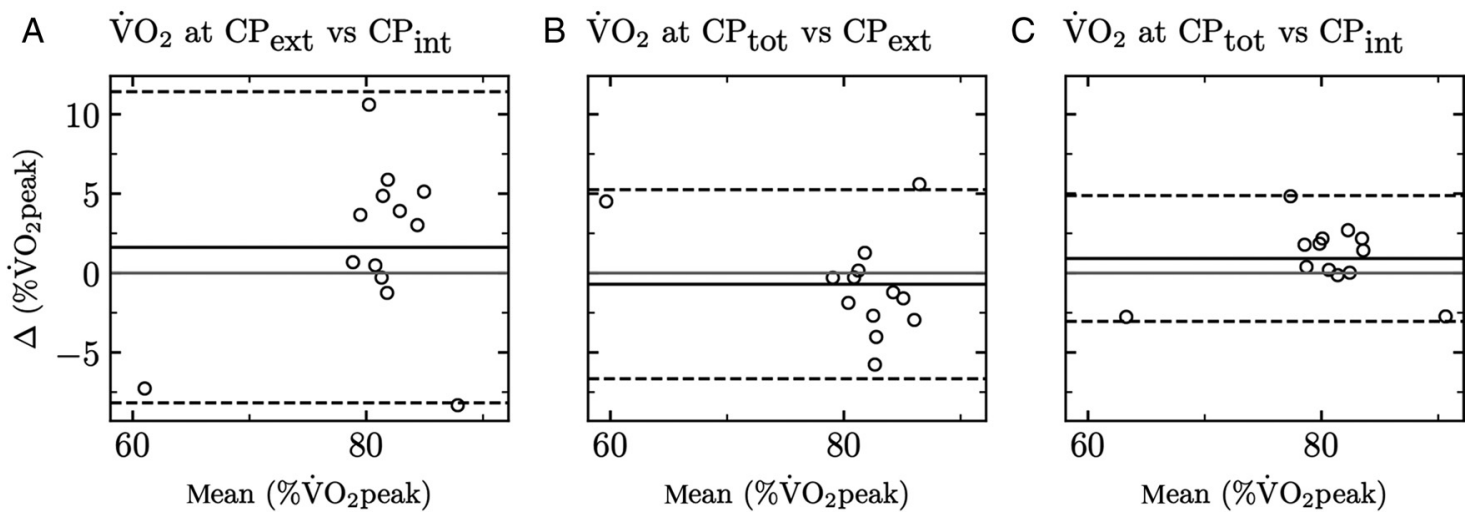

Figure 3 - Bland-Altman plots comparing $\dot{\mathrm{VO}} 2$ (expressed as a $\% \dot{\mathrm{VO}} \mathrm{O}_{2}$ peak) between $(\mathrm{A}) \mathrm{CP}_{\text {ext }}$ and $\mathrm{CP}_{\text {int }},(\mathrm{B}) \mathrm{CP}_{\text {tot }}$ and $\mathrm{CP}_{\text {ext }}$, and $(\mathrm{C}) \mathrm{CP}_{\text {tot }}$ and $\mathrm{CP}_{\text {int }}$. $\mathrm{CP}$ indicates critical power; $\mathrm{CP}_{\text {ext }}$, external $\mathrm{CP} ; \mathrm{CP}_{\text {int }}$, internal $\mathrm{CP} ; \mathrm{CP}_{\text {tot }}$, total $\mathrm{CP} ; \dot{\mathrm{VO}}_{2}$, oxygen uptake; $\% \dot{\mathrm{VO}} 2$ peak, percentage of peak rate of oxygen uptake.

\begin{abstract}
Table 1 Mean (SD) of CS Estimated Using Time to Exhaustion and $\mathrm{CP}_{\text {ext }}, \mathrm{CP}_{\text {int }}$, and $\mathrm{CP}_{\text {tot }}$ Estimated Using Strides Recorded During First (Start), Middle (Half), and Last (End) Minute of Each Time to Exhaustion, As Well As $\mathrm{VO}_{2}$ (Expressed as a $\% \dot{\mathrm{VO}}_{2}$ peak) at the CS/CPs
\end{abstract}

\begin{tabular}{lcc}
\hline $\begin{array}{l}\text { Critical } \\
\text { intensity }\end{array}$ & $\begin{array}{c}\text { Critical intensity value, } \\
\mathbf{m} / \mathbf{s} \text { or } \mathbf{W} / \mathbf{k g}\end{array}$ & $\dot{\mathbf{V O}}_{\mathbf{2}}, \% \mathbf{V}_{\mathbf{2}}$ peak \\
\hline $\mathrm{CS}$ & $4.27(0.40)$ & $82.5(5.3)$ \\
$\mathrm{CP}_{\text {ext }}$ & & \\
Start & $5.16(0.56)$ & $81.3(7.6)$ \\
Half & $5.26(0.46)$ & $82.6(5.2)$ \\
End & $5.28(0.49)$ & $83.1(6.0)$ \\
$\mathrm{CP}_{\text {int }}$ & & \\
Start & $2.60(0.54)$ & $79.7(6.0)$ \\
Half & $2.53(0.62)$ & $78.9(6.9)$ \\
End & $2.53(0.43)$ & $78.9(4.2)$ \\
$\mathrm{CP}_{\text {tot }}$ & & \\
Start & $7.78(1.01)$ & $80.6(6.3)$ \\
Half & $7.77(0.94)$ & $80.5(5.6)$ \\
End & $7.81(0.79)$ & $80.8(3.9)$ \\
\hline
\end{tabular}

Abbreviations: $\mathrm{CP}$, critical power; $\mathrm{CP}_{\text {ext }}$, external $\mathrm{CP}$; $\mathrm{CP}_{\text {int }}$, internal $\mathrm{CP} ; \mathrm{CP}_{\text {tot }}$, total CP; CS, critical speed; RM-ANOVA, repeated-measures analysis of variance; $\dot{\mathrm{V}} \mathrm{O}_{2}$, oxygen uptake; $\% \dot{\mathrm{V}} \mathrm{O}_{2}$ peak, percentage of peak rate of oxygen uptake. Note: One-way RM-ANOVA reported no significant difference between the $\mathrm{VO}_{2}$ for CS and CPs at the start $(P=.09)$. One-way RM-ANOVA reported no significant main effect of the time stride selection when estimating $\mathrm{CP}_{\mathrm{ext}}, \mathrm{CP}_{\text {int }}$, and $\mathrm{CP}_{\text {tot }}$, as well as for the resulting $\mathrm{V}_{2}(P \geq .44)$.

necessarily correspond to the same specific intensity threshold for everyone.

On the other hand, consistent $\dot{\mathrm{VO}}_{2}$ were obtained among the 3 different CPs (Table 1), and no systematic biases were reported (Figure 3), which proved that using $P_{\text {ext }}, P_{\text {int }}$, and $P_{\text {tot }}$ to estimate corresponding CPs led to similar $\mathrm{VO}_{2}$. Our findings corroborate those of Barker et al, ${ }^{22}$ who report that $\mathrm{CP}$ represents a specific $\dot{\mathrm{V}} \mathrm{O}_{2}$, which can be achieved at different combinations of $P_{\text {ext }}$ and $P_{\text {int }}$ (pedaling frequencies) outputs in cycling. Therefore, the present study is the first to show that $\mathrm{VO}_{2}$ at $\mathrm{CP}$ represents a specific metabolic rate independent of the type of power output considered (internal vs external vs total) in running. Practically, this means that if one is only interested in the $\mathrm{VO}_{2}$ associated with $\mathrm{CP}$, it can be obtained simply by using an inertial sensor. Indeed, this sensor would provide the spatiotemporal parameters required to calculate $P_{\text {int }}$ during the 4 exhaustive runs, that is, ground contact time, stride frequency, and duty factor. These $P_{\text {int }}$ s would then be used to estimate $\mathrm{CP}_{\text {int }}$, which would finally be matched to the $P_{\mathrm{int}} \mathrm{s}$ computed during the maximal incremental aerobic test to obtain the $\dot{\mathrm{VO}}_{2}$ associated to $\mathrm{CP}_{\text {int }}$. Therefore, this would avoid the need for an expensive instrumented treadmill as well as the more complicated analysis of the ground reaction force to obtain $P_{\text {ext }}$ to estimate $\mathrm{CP}_{\text {ext }}$. However, typical power meters ${ }^{19}$ provide $P_{\text {ext }}$ or $P_{\text {tot }}$ but not $P_{\text {int }}$, which makes the estimation of $\mathrm{CP}_{\text {ext }}$ or $\mathrm{CP}_{\text {tot }}$ essential to perform training based on power-based intensity domains. Besides, Vassallo et al ${ }^{34}$ recently estimated a running $\mathrm{CP}_{\text {tot }}$ from a 3-minute all-out test performed on an outdoor athletic track. The authors obtained a $\mathrm{CP}_{\text {tot }}$ of $6.64 \mathrm{~W} / \mathrm{kg}$ (assuming a body mass of $68 \mathrm{~kg}$ ), which is $15 \%$ smaller than the one reported in the present study. Nonetheless, the difference might be explained by the different methodology employed: $\mathrm{CP}_{\text {tot }}$ was given by the running power averaged during the last 30 seconds of the 3-minute test and running power was computed from speed data recorded using a global positioning system sampling at $10 \mathrm{~Hz}$. Moreover, participants were less trained, that is, they reported a $20 \%$ smaller $\dot{\mathrm{V}}{ }_{2}$ peak $(51.1 \mathrm{~mL} / \mathrm{kg} / \mathrm{min})$ than in the present study, which obviously lead to a smaller CP.

No effect of time stride selection was obtained when estimating $\mathrm{CP}_{\text {ext }}, \mathrm{CP}_{\text {int }}$, and $\mathrm{CP}_{\text {tot }}$, as well as for their resulting $\dot{\mathrm{V}} \mathrm{O}_{2}$ (Table 1). Indeed, using the first, half, or last minute of the exhaustive runs to calculate $P_{\mathrm{ext}}, P_{\mathrm{int}}$, and $P_{\text {tot }}$ were equivalent, except for the $110 \%$ PS intensity, though the difference could be assumed negligible $(0.15 \mathrm{~W} / \mathrm{kg}$, ie, $10 \mathrm{~W}$ for a $70-\mathrm{kg}$ person; Table 2). Therefore, using the average of the powers calculated at the first, half, and last minute of the exhaustive runs would have led to similar estimations of CPs than when using these time stride selections separately. Besides, the similar $P_{\text {ext }}, P_{\text {int }}$, and $P_{\text {tot }}$ obtained in this study within each exhaustive run and independently of the intensity of the run (submaximal or supramaximal) is consistent with the observations of Avogadro et al, ${ }^{27}$ which depicted similar $W_{\text {ext }}, W_{\text {int }}$, and $W_{\text {tot }}$ at the third and last minute of an exhaustive run at a speed corresponding to $90 \% \mathrm{VO}_{2} \max$ and with similar $W_{\text {ext }}$ at the end of an exhaustive run at $95 \% \dot{\mathrm{VO}}_{2} \max$ than at the beginning of the slow component of $\mathrm{VO}_{2}(\sim 120 \mathrm{~s}) .^{24}$ However, these results disagreed with the fact that the same 
Table 2 Mean (SD) of the Time to Exhaustion Corresponding to the 4 Exhaustive Runs Performed at $90 \%, 100 \%$, $110 \%$, and $120 \%$ of the Participant's PS, As Well As $P_{\text {ext }}, P_{\text {int }}$, and $P_{\text {tot }}$ Power Computed Using Strides Recorded During the First (Start), Middle (Half), and Last (End) Minute of Each Time to Exhaustion

\begin{tabular}{lccc}
\hline Running speed (\%PS) & $\mathbf{9 0}$ & $\mathbf{1 0 0}$ & $\mathbf{1 1 0}$ \\
\hline Time to exhaustion (min) & $15.25(2.50)$ & $5.68(1.10)$ & $2.65(0.78)$ \\
$P_{\text {ext }}, \mathrm{W} / \mathrm{kg}$ & & & 120 \\
$\quad$ Start & $5.77(0.35)$ & $6.38(0.44)$ & $6.94(0.43)$ \\
Half & $5.74(0.39)$ & $6.29(0.42)$ & $6.90(0.43)$ \\
End & $5.77(0.37)$ & $6.32(0.39)$ & $6.95(0.40)$ \\
$P_{\text {int }}$ W/kg & & & $7.56(0.45)$ \\
Start & $3.26(0.44)$ & $4.12(0.58)$ & $5.14(0.72)$ \\
Half & $3.26(0.42)$ & $4.15(0.56)$ & $5.19(0.71)$ \\
End & $3.24(0.41)$ & $4.16(0.56)$ & $5.28(0.71)$ \\
$P_{\text {tot }}$ W/kg & & & $6.39(0.99)$ \\
Start & $9.03(0.73)$ & $10.50(0.96)$ & $6.41(0.90)$ \\
Half & $9.00(0.74)$ & $10.44(0.91)$ & $6.51(0.92)$ \\
End & $9.02(0.69)$ & $10.48(0.85)$ & $12.08(1.06) *$ \\
\end{tabular}

Abbreviations: $P$, power; $P_{\text {ext }}$, external P; $P_{\text {int }}$, internal P; PS, peak speed; $P_{\text {tot }}$, total $P$; RM-ANOVA, repeated-measures analysis of variance. Note: Two-way RM-ANOVA reported no significant main effect of the time stride selection on the calculation of $P_{\mathrm{ext}}, P_{\mathrm{int}}$, and $P_{\mathrm{tot}}(P \geq .11)$, while there was a significant main effect of $\%$ PS $(P<.001)$, with each of the 3 powers at \%PS being statistically higher compared with those at previous \%PS, as reported by Holm post hoc tests $(P<.001)$. Significant differences as determined by post hoc tests for the time stride selection $\times \%$ PS interaction effect obtained for $P_{\text {tot }}(P=.03)$ : *significantly different from the end at $110 \%$ PS. Note that, a statistically higher $P_{\text {tot }}$ was obtained for all comparisons between an higher \%PS and a lower one $(P<.001 ; 54$ comparisons $)$ but was not represented by a symbol in the table.

authors ${ }^{24}$ as well as Candau et $\mathrm{al}^{26}$ observed a lower $W_{\text {int }}$ at the end of the exhaustive run than at its beginning and that a larger $W_{\text {ext }}$ was obtained right before exhaustion. ${ }^{26}$ Although these discrepancies with previous findings may be due to methodological differences associated with the device used to assess the mechanical power output (kinematic arm vs instrumented treadmill) and the running speed tested (submaximal vs supramaximal), the present study depicted no effect of the time stride selection when calculating running powers at intensities ranging from 90\%PS to $120 \% \mathrm{PS}$. Thus, these results further extended the knowledge of the effect of the time stride selection on power calculations, especially at supramaximal intensities.

\section{Practical Applications}

Power is becoming a widely used external metric in running, and is especially useful when speed is no longer a relevant metric to separate between intensity domains (running on a variable terrain or in a very windy condition). In such case, commercial systems (eg, Runscribe, Stryd, or Myotest) provide coaches and athletes an easy-to-use tool to monitor running power. ${ }^{19}$ Thereby, combining this outcome with the knowledge of $\mathrm{CP}$ could allow athletes to control their targeted training intensity and coaches to prescribe a training session in any type of condition.

\section{Conclusions}

To conclude, the present study estimated the usual CS as well as $\mathrm{CP}_{\text {ext }}, \mathrm{CP}_{\text {int }}$, and $\mathrm{CP}_{\text {tot }}$ in running. The $\dot{\mathrm{VO}}_{2}$ at $\mathrm{CS}$ and $\mathrm{CPs}$ were not statistically different. No systematic bias was reported between the $\dot{\mathrm{V}} \mathrm{O}_{2}$ at $\mathrm{CS}$ and $\mathrm{CP}_{\text {ext }}$ as well as among the $\dot{\mathrm{V}} \mathrm{O}_{2}$ at the 3 different CPs, whereas systematic biases were obtained among the $\dot{\mathrm{VO}}_{2}$ at $\mathrm{CS}$ and internal and total CP. Nonetheless, these differences were small $(\leq 3 \%)$ and thus not clinically relevant. Therefore, these results suggest that $\mathrm{CS}$ and $\mathrm{CPs}$ closely represent the same fatigue threshold in running. Furthermore, this study reported no effect of the time stride selection when calculating $P_{\text {ext }}, P_{\text {int }}$, and $P_{\text {tot }}$, when estimating $\mathrm{CP}_{\text {ext }}, \mathrm{CP}_{\text {int }}$, and $\mathrm{CP}_{\text {tot }}$, as well as when calculating their resulting $\mathrm{VO}_{2}$, which further extends the knowledge of the effect of the time stride selection on power calculations.

\section{Acknowledgments}

This study was supported by the University of Lausanne (Switzerland). The authors warmly thank the participants for their time and cooperation.

\section{References}

1. Garber CE, Blissmer B, Deschenes MR, et al. American College of Sports Medicine position stand. Quantity and quality of exercise for developing and maintaining cardiorespiratory, musculoskeletal, and neuromotor fitness in apparently healthy adults: guidance for prescribing exercise. Med Sci Sports Exerc. 2011;43(7):1334-1359. PubMed ID: 21694556 doi:10.1249/MSS.0b013e318213fefb

2. Sjödin B, Svedenhag J. Applied physiology of marathon running. Sports Med. 1985;2(2):83-99. PubMed ID: 3890068 doi:10.2165/ 00007256-198502020-00002

3. Billat LV, Koralsztein JP. Significance of the velocity at VO2max and time to exhaustion at this velocity. Sports Med. 1996;22(2):90-108. PubMed ID: 8857705 doi:10.2165/00007256-199622020-00004

4. Swain DP, Leutholtz BC, King ME, Hass LA, Branch JD. Relationship between \% heart rate reserve and \% VO2 reserve in treadmill exercise. Med Sci Sports Exerc. 1998;30(2):318-321. PubMed ID: 9502363 doi:10.1097/00005768-199802000-00022

5. Scharhag-Rosenberger F, Meyer T, Gabler N, Faude O, Kindermann $\mathrm{W}$. Exercise at given percentages of $\mathrm{VO} 2 \mathrm{max}$ : heterogeneous metabolic responses between individuals. J Sci Med Sport. 2010;13(1): 74-79. PubMed ID: 19230766 doi:10.1016/j.jsams.2008.12.626 
6. Lansley KE, Dimeena FJ, Bailey SJ, Jones AM. A 'new' method to normalise exercise intensity. Int J Sports Med. 2011;32(7):535-541. PubMed ID: 21563028 doi:10.1055/s-0031-1273754

7. Iannetta D, Inglis EC, Mattu AT, et al. A critical evaluation of current methods for exercise prescription in women and men. Med Sci Sports Exerc. 2020;52(2):466-473. PubMed ID: 31479001 doi:10.1249/ MSS.0000000000002147

8. Burnley M, Jones AM. Oxygen uptake kinetics as a determinant of sports performance. Eur J Sport Sci. 2007;7(2):63-79. doi:10.1080/ 17461390701456148

9. Whipp BJ, Mahler M. Dynamics of pulmonary gas exchange during exercise. In: West JB ed. Pulmonary gas exchange. Vol. 2. New York, NY: Academic Press; 1980.

10. Wasserman K, Whipp BJ, Koyl SN, Beaver WL. Anaerobic threshold and respiratory gas exchange during exercise. J Appl Physiol. 1973; 35(2):236-243. PubMed ID: 4723033 doi:10.1152/jappl.1973.35. 2.236

11. Vanhatalo A, Doust AH, Burnley M. Determination of critical power using a 3-min all-out cycling test. Med Sci Sports Exerc. 2007; 39(3):548-555. PubMed ID: 17473782 doi:10.1249/mss.0b013e31 $802 \mathrm{dd} 3 \mathrm{e} 6$

12. Jones AM, Burnley M, Black MI, Poole DC, Vanhatalo A. The maximal metabolic steady state: redefining the 'gold standard.' Physiol Rep. 2019;7(10):e14098. PubMed ID: 31124324 doi:10. 14814/phy2.14098

13. Monod H, Scherrer J. The work capacity of a synergic muscular group. Ergonomics. 1965;8(3):329-338. doi:10.1080/0014013650 8930810

14. Poole DC, Ward SA, Gardner GW, Whipp BJ. Metabolic and respiratory profile of the upper limit for prolonged exercise in man. Ergonomics. 1988;31(9):1265-1279. PubMed ID: 3191904 doi:10.1080/00140138808966766

15. Jones AM, Vanhatalo A, Burnley M, Morton RH, Poole DC. Critical power: implications for determination of $\mathrm{V}^{\cdot} \mathrm{O} 2 \mathrm{max}$ and exercise tolerance. Med Sci Sports Exerc. 2010;42(10):1876-1890. PubMed ID: 20195180 doi:10.1249/MSS.0b013e3181d9cf7f

16. Craig JC, Vanhatalo A, Burnley M, et al. Critical power: Possibly the most important fatigue threshold in exercise physiology. In: Zoladz JA, ed. Muscle and Exercise Physiology. Academic Press; 2019: 159-181.

17. Jones AM, Vanhatalo A. The 'critical power' concept: applications to sports performance with a focus on intermittent high-intensity exercise. Sports Med. 2017;47(suppl 1):65-78. doi:10.1007/s40279-0170688-0

18. Morton RH, Hodgson DJ. The relationship between power output and endurance: a brief review. Eur J Appl Physiol Occup Physiol. 1996;73(6):491-502. PubMed ID: 8817118 doi:10.1007/BF00 357670

19. Jaén-Carrillo D, Roche-Seruendo LE, Carton-Llorente A, RamirezCampillo R, Garcia-Pinillos F. Mechanical power in endurance running: a scoping review on sensors for power output estimation during running. Sensors. 2020;20(22):6482. doi:10.3390/s20226482
20. Cavagna GA. Force platforms as ergometers. J Appl Physiol. 1975; 39(1):174-179. PubMed ID: 1150585 doi:10.1152/jappl.1975.39. 1.174

21. Nardello F, Ardigò LP, Minetti AE. Measured and predicted mechanical internal work in human locomotion. Hum Mov Sci. 2011;30(1): 90-104. PubMed ID: 21056491 doi:10.1016/j.humov.2010.05.012

22. Barker T, Poole DC, Noble ML, Barstow BJ. Human critical poweroxygen uptake relationship at different pedalling frequencies. Exp Physiol. 2006;91(3):621-632. PubMed ID: 16527863 doi:10.1113/ expphysiol.2005.032789

23. Smith JC, Hill DW. Stability of parameter estimates derived from the power/time relationship. Can J Appl Physiol. 1993;18(1):43-47. PubMed ID: 8471993 doi:10.1139/h93-005

24. Borrani F, Candau R, Perrey S, Millet GY, Millet GP, Rouillon J-D. Does the mechanical work in running change during the $\mathrm{VO}_{2}$ slow component? Med Sci Sports Exerc. 2003;35(1):50-57. PubMed ID: 12544635 doi:10.1097/00005768-200301000-00009

25. Clark IE, Vanhatalo A, Thompson P, et al. Dynamics of the powerduration relationship during prolonged endurance exercise and influence of carbohydrate ingestion. J Appl Physiol. 2019;127(3):726736. PubMed ID: 31295069 doi:10.1152/japplphysiol.00207.2019

26. Candau R, Belli A, Millet GY, Geroges D, Barbier B, Rouillon JD. Energy cost and running mechanics during a treadmill run to voluntary exhaustion in humans. Eur J Appl Physiol Occup Physiol. 1998; 77(6):479-485. PubMed ID: 9650730 doi:10.1007/s004210050363

27. Avogadro P, Dolenec A, Belli A. Changes in mechanical work during severe exhausting running. Eur J Appl Physiol. 2003;90(1-2): 165-170. PubMed ID: 14504949 doi:10.1007/s00421-003-0846-y

28. Kuipers H, Rietjens G, Verstappen F, Schoenmakers H, Hofman G. Effects of stage duration in incremental running tests on physiological variables. Int J Sports Med. 2003;24(7):486-491. PubMed ID: 12968205 doi:10.1055/s-2003-42020

29. Morton HR. A 3-parameter critical power model. Ergonomics. 1996; 39(4):611-619. PubMed ID: 8854981 doi:10.1080/001401396089 64484

30. Patoz A, Spicher R, Pedrani N, Malatesta D, Borrani F. Critical speed estimated by statistically appropriate fitting procedures. Eur J Appl Physiol. 2021;121(7):2027-2038.

31. Levenberg K. A method for the solution of certain non-linear problems in least squares. Q Appl Math. 1944;2(2):164-168. doi:10.1090/ qam/10666

32. Marquardt DW. An algorithm for least-squares estimation of nonlinear parameters. J Soc Ind Appl Math. 1963;11(2):431-441. doi:10. $1137 / 0111030$

33. Jones AM, Vanhatalo A, Burnelu M, Morton RH, Poole DC. Response. Med Sci Sports Exerc. 2011;43(3):553. doi:10.1249/ MSS.0b013e3182075485

34. Vassallo C, Gray A, Cummins C, Murphy A, Waldron M. Exercise tolerance during flat over-ground intermittent running: modelling the expenditure and reconstitution kinetics of work done above critical power. Eur J Appl Physiol. 2020;120(1):219-230. PubMed ID: 31776696 doi:10.1007/s00421-019-04266-8 